\title{
Liver Transplantation in Acute-on-Chronic Liver Failure: Considerations for a Systematic Approach to Decision Making
}

\author{
Michael Heise $^{\mathrm{a}} \quad$ Nina Weiler $^{\mathrm{b}}$ Sonja Iken ${ }^{\mathrm{c}} \quad$ Martin-Walter Welker $^{\mathrm{b}} \quad$ Stefan Zeuzem $^{\mathrm{b}}$ \\ Wolf O. Bechstein ${ }^{a}$ Andreas A. Schnitzbauer ${ }^{a}$ \\ ${ }^{a}$ Clinic for General and Visceral Surgery, University Hospital Frankfurt, Goethe-University Frankfurt, Frankfurt/M., Germany; \\ ${ }^{b}$ Clinic for Medicine I, University Hospital Frankfurt, Goethe-University Frankfurt, Frankfurt/M., Germany; \\ ${ }^{\mathrm{c}}$ Clinic for Anesthesiology, Intensive Care and Pain Care, University Hospital Frankfurt, Goethe-University Frankfurt, Frankfurt/M., Germany
}

\section{Keywords}

Acute-on-chronic liver failure - Liver transplantation . Kidney function

\section{Summary}

Background: Acute-on-chronic liver failure (ACLF) is a complex disease with deteriorating liver and kidney function and associated organ failure in patients with chronic liver disease. Methods: This is a concise overview for bedside and algorithmic decision making in patients with ACLF based on the most recent literature. Results: Diagnosis and dynamics of ACLF can be easily monitored with the CLIF-C-ACLF calculator, which delivers grading for ACLF and estimates the risk of mortality, as the natural transplant-free course of ACLF is often fatal. Transplantation offers the best results in patients with ACLF that do not recover spontaneously. However, marginal donor organs should be avoided. Conclusion: ACLF is an increasingly relevant indication with good outcome after liver transplantation. Adequate donor rates may reduce the incidence by means of timely transplantation of acute decompensated patients in lower stages of urgency. Future challenges comprise specific allocation of donor organs to this group of patients that are at a similar risk of mortality when compared to acute liver failure.

(C) 2018 S. Karger GmbH, Freiburg

\section{Introduction and Rationale}

Acute-on-chronic liver failure (ACLF) is a heterogeneous disease that is characterized by an acute deterioration of liver function in patients with a cirrhotic predisposition following an injury like infection or substance abuse. This may result in a systemic response of inflammation and lead to (multiple) organ failure (PIRO concept) [1]. Focusing on the last 6 years of evidence, this article provides a concise overview and summary of the literature on liver transplantation in patients with ACLF. This summary will provide specific evidence and considerations in order to reach a decision for or against liver transplantation, including diagnosing ACLF, risk estimation in indicating liver transplantation (ideal timing vs. futility of care), as well as an outlook for future allocation challenges in patients awaiting liver transplantation for ACLF.

\section{Characteristics of ACLF}

Already in 2012 the European Association for the Study of the Liver (EASL) characterized ACLF based on the PIRO concept: patients with cirrhosis that experience an injury like bacterial infection [2] or translocation react with a deterioration of their liver function, leading to immune paralysis, sepsis, and multi-organ failure (MOF). In the worst case this cascade is irreversible and leads to death [1]. Just 1 year later, Moreau et al. [3] published the results of the CANONIC trial in which they defined diagnostic criteria for ACLF. Basically, they analyzed $>1,200$ patients with liver cirrhosis and acute decompensation and identified prognostic factors defining ACLF. These factors were supposed to help in distinguishing patients with ACLF from those with just acute decompensation. The majority of patients without ACLF $(>1,000)$ had a transplant-

\section{KARGER}

() 2018 S. Karger GmbH, Freiburg
Prof. Dr. Andreas A. Schnitzbauer, MD, FACS, FEBS 


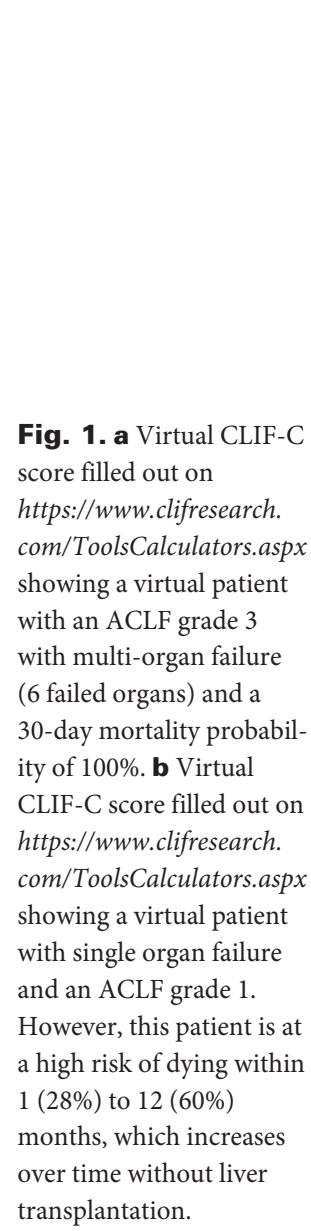

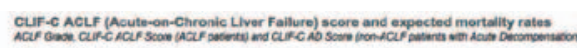

$1 a$
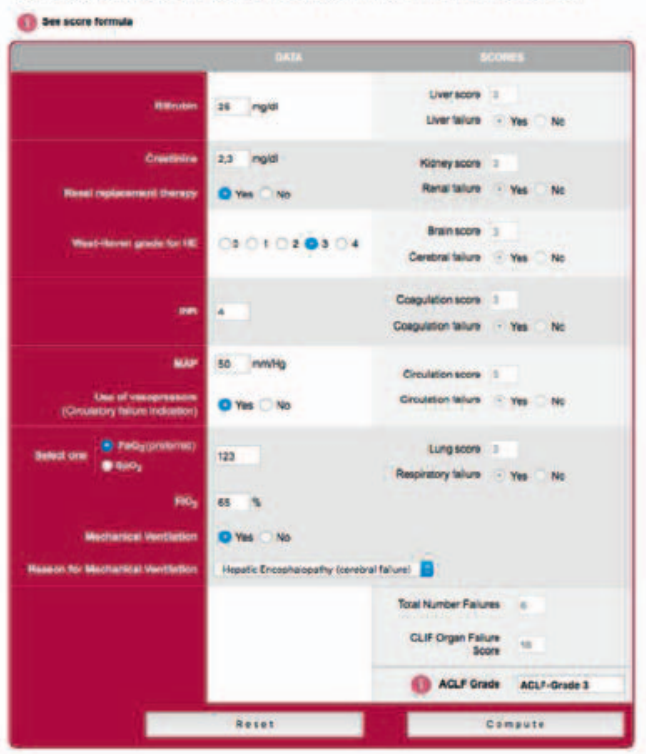

Probability of dying

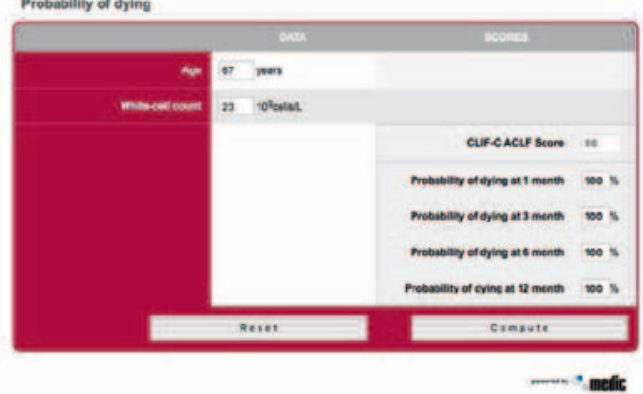

$1 b$
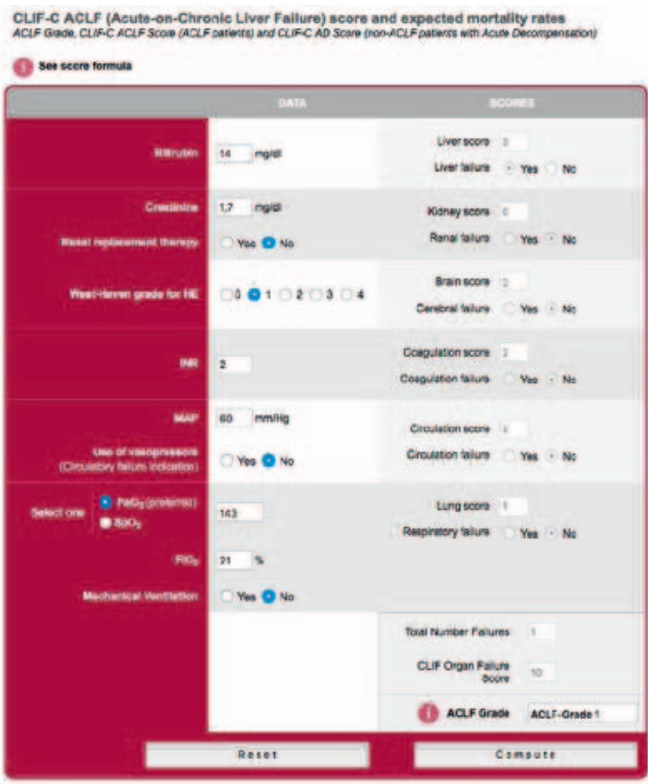

Probability of dying

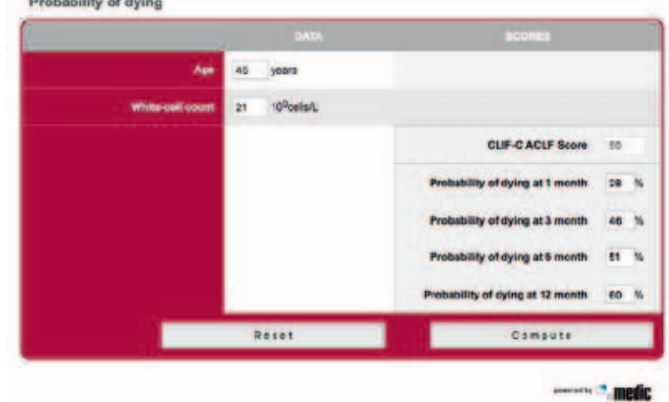

free 28-day and 90-day mortality of 4.7 and 14\%, respectively, which is characterized by the absence of creatinine values $>1.5 \mathrm{mg} /$ $\mathrm{dl}$ and the absence of organ failure or single-non-kidney organ failure. The patients with ACLF were classified into three categories:

- ACLF grade 1: single organ kidney failure; or single failure of the liver, coagulation, respiration, and elevated creatinine of $1.5-1.9 \mathrm{mg} / \mathrm{dl}$ or hepatic encephalopathy (HE) [4] grade I or II; or HE grade III/IV and elevated creatinine of $1.5-1.9 \mathrm{mg} / \mathrm{dl}$;

- ACLF grade 2: 2 organ failures;

- ACLF grade 3: $\geq 3$ organ failures.

The corresponding liver transplant-free 90 -day mortality rates increased from 40.7 to 52.3 to $79.1 \%$ [3].

\section{Prognosis and Risk Estimation}

The prognosis of ACLF is characterized by high mortality, although reversibility is possible by multi-organ supportive care and a recommended best practice protocol-based approach $[5,6]$. This is based on the experience gained from the treatment of acute liver failure, which is similar to ACLF in multiple ways. Recommendations imply a multidisciplinary setting including psychological support, rehabilitation, and an early ICU(intensive care unit)-based treatment with early specific organ support maintaining the physiologic homeostasis rather than correcting it. This includes early intubation in patients with HE to prevent aspiration. Furthermore, infection should be treated in a targeted way, utilizing repeat cultures and avoiding the uncritical application of broad-spectrum antibiotics [6-8]. Risk estimation is possible with the CLIF-C-ACLF calculator that reflects organ failure (liver, kidney, brain, circulation, lung) as well as delivers the ACLF grade and thereafter includes age and white blood cell count into the formula, finally delivering probabilities for 1-month to 1-year mortality with non-liver transplant treatment (www.clifresearch.com) (fig. 1) [9]. The clinical superiority of the CLIF-C-ACLF scores should be compared to other scores such as the MELD (model for end-stage liver disease) score. However, the severity and prognosis (mortality) were not related to the precipitating event in the CANONIC trial and the initial ACLF grade is basically not the final grade in more than $80 \%$ of patients. Therefore, the grading should be repeated until day $3-7$ when the grade stabilizes and a more reliable prognostic estimation can be made $[3,10]$.

\section{The Golden Window for Liver Transplantation versus Futility of Care}

Altogether, this leads to the conception of the golden window of ACLF in which a liver transplantation is the optimal therapeutic op- 
Fig. 2. Diagram of the conception of the golden window for transplantation (modified from Reddy et al. [6]).

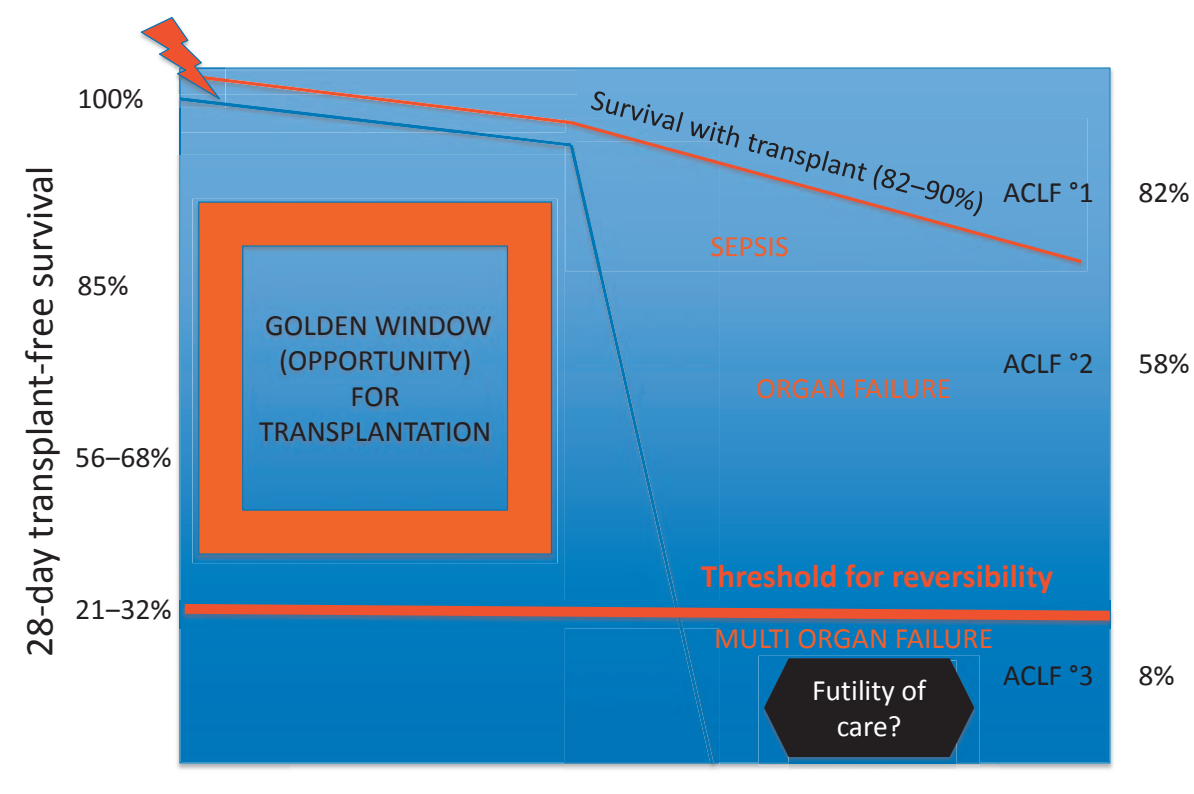

Time, months tion (fig. 2). The median transplant-free survival in patients with ACLF is 48 days. As outlined above, the ACLF grading is terminated and varies only slightly after days $3-7$. Scores 1 and 2 promise posttransplant survival rates of $82-90 \%$ after 1 year. However, an ongoing vicious circle of sepsis and (multi-)organ failure leads to a higher risk of mortality and irreversibility. The deterioration and worsening into ACLF grade 3 closes the window for transplantation due to chronic MOF. Thereafter, evidence shows futility of care and irreversibility with a mortality of $>80 \%$ in all patients with ACLF grade 3 , which applies for approximately $8 \%$ of all patients with ACLF [6].

\section{Outcomes of Liver Transplantation after Acute-on-Chronic Liver Failure}

To date, there are two outstanding reports highlighting liver transplantation for ACLF. Finkenstedt et al. [11] analyzed 144 patients with ACLF. Of those, 94 were evaluated for transplantation, 77 were listed, and 33 were finally transplanted. Notably, of the 77 listed patients 36 died on the waiting list and 2 were withdrawn for other reasons. Of the 111 patients that did not undergo liver transplantation only 10 were alive, which accounts for a 1-year mortality risk of $90 \%$. The survival rates for the patients undergoing transplantation at 1 year and 5 years were 87 and $82 \%$, respectively, reflecting similar outcomes as in patients undergoing liver transplantation for other indications [11]. Artru et al. [12] obtained similar results in their recent report. They analyzed over 1,500 liver transplantations over 7 years. ACLF grade I was present in 119, grade II in 145 , and grade III in 73 patients. The 1-year overall survival was $82-86 \%$, and the MELD score ranged between 29 and 38. Notably, grade III patients had a $100 \%$ complication rate after liver transplantation and the longest stay (51 days) [12].

\section{Implication of Allocation Rules for ACLF Considering Waiting List Mortality and Economic Aspects}

Based on the high mortality risk, there has to be a debate about prioritization in patients with ACLF on the waiting list for liver transplantation based on the existing evidence and allocation rules. MELD scores of 35-40 and higher are stringently and significantly associated with excess mortality on the waiting list and show comparable outcomes to high urgent patients $[13,14]$. There is a lack of treatment alternatives, and the natural course on ICUs is often lethal $[3,5,6]$. Besides a super-urgent status, an alternative option may be local allocation of organs in these patients. This reduces cold ischemia times and rewards high efforts and well-structured donor regions (quality of structure) by regional allocation. This would indirectly reward donors and donor families by supplying the most urgent patients within the golden window of ACLF and the region of donation. Low ischemic times, low donor age, and whole organ transplants are conditions for good outcome after liver transplantation in ACLF. Since the postoperative course is associated with more complications and inferior outcome when compared to other indications for liver transplantation and the economic burden may be higher in this group of patients with otherwise very limited chance of survival, these aspects warrant special attention $[5,6,8,15-17]$.

\section{Checklist and Algorithm for Decision Making}

Figure 3 proposes a checklist and algorithm for daily decision making in patients with ACLF. Basically, it summarizes the factors outlined above. Daily re-evaluation, decision making up to day 3-7 for $80 \%$ of patients based on objective criteria and utilizing prog- 


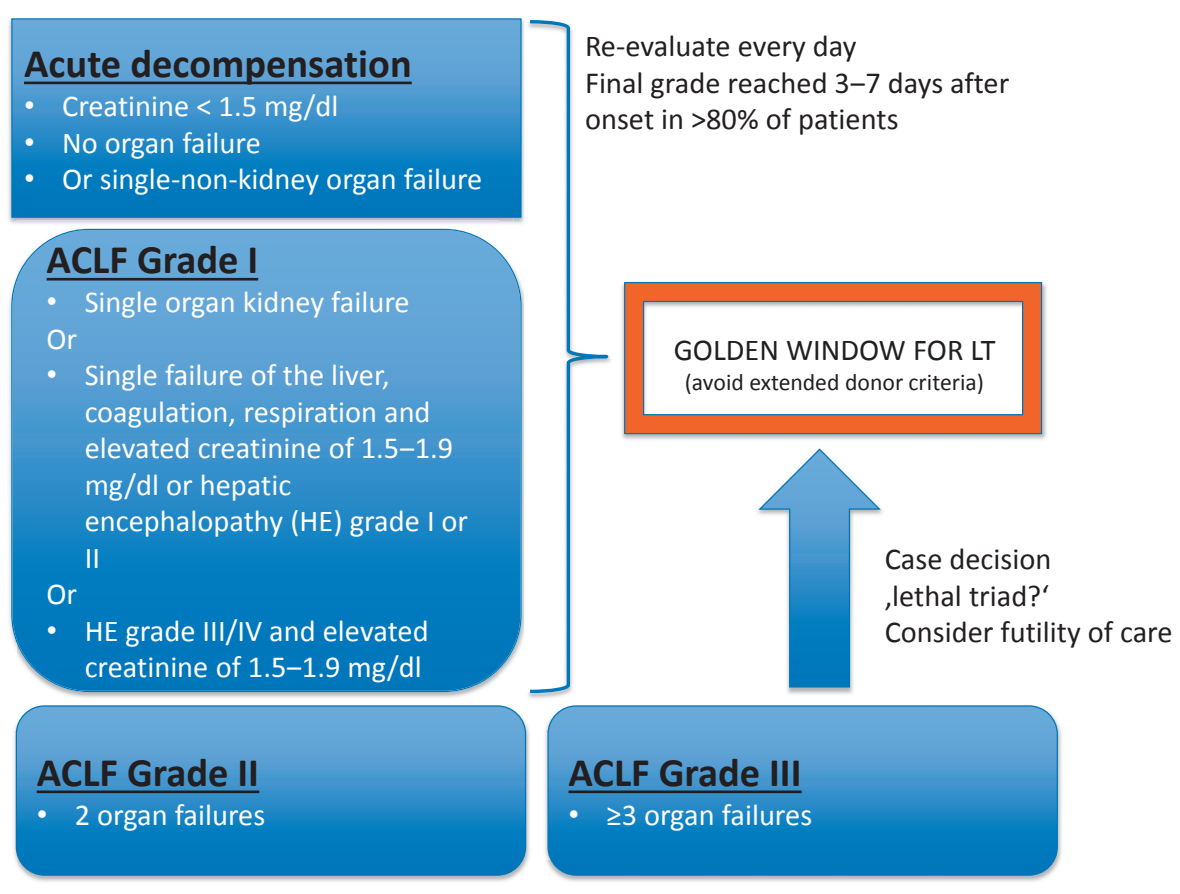

Fig. 3. Proposal for an algorithm for simplified decision making in patients with acute decompensation or acute-on-chronic liver failure (ACLF) based on grading of ACLF and considering dynamics of ACLF grading 3-7 days after onset.

nostic calculators and clinical judgment with attention to detail, and a strict continuity of care help to select those patients best mental condition for adequate supply of organs in an optimal timely manner. suited for transplantation. Nonetheless, a fundamental condition for beneficial outcome in super-urgent cases is a sufficient supply with donor organs in a timely and adequate manner. Donors and their relatives enable the success of transplantation. They donate their life-saving organs after irreversible failure of the brain or after

\section{Disclosure Statement}

The authors do not have any conflicts of interest.

\section{References}

1 Jalan R, Gines P, Olson JC, et al: Acute-on chronic liver failure. J Hepatol 2012;57:1336-1348.

2 Bajaj JS, O'Leary JG, Reddy KR, et al: Survival in infection-related acute-on-chronic liver failure is defined by extrahepatic organ failures. Hepatol Baltim Md 2014; 60:250-256.

3 Moreau R, Jalan R, Gines P, et al: Acute-on-chronic liver failure is a distinct syndrome that develops in patients with acute decompensation of cirrhosis. Gastroenterology 2013;144:1426-1437, 1437-1439.

4 Vilstrup H, Amodio P, Bajaj J, et al: Hepatic encephalopathy in chronic liver disease: 2014 Practice Guideline by the American Association for the Study of Liver Diseases and the European Association for the Study of the Liver. Hepatol Baltim Md 2014;60:715-735.

5 Bernal W, Jalan R, Quaglia A, et al: Acute-on-chronic liver failure. Lancet Lond Engl 2015;386:1576-1587.

6 Reddy MS, Rajalingam R, Rela M: Liver transplantation in acute-on-chronic liver failure: lessons learnt from acute liver failure setting. Hepatol Int 2015;9: 508-513.
McPhail MJW, Shawcross DL, Abeles RD, et al: Increased survival for patients with cirrhosis and organ failure in liver intensive care and validation of the chronic liver failure-sequential organ failure scoring system. Clin Gastroenterol Hepatol 2015;13:13531360.e8.

7 Bernal W, Hyyrylainen A, Gera A, et al: Lessons from look-back in acute liver failure? A single centre experience of 3300 patients. J Hepatol 2013;59:74-80.

$\checkmark$ Jalan R, Saliba F, Pavesi M, et al: Development and validation of a prognostic score to predict mortality in patients with acute-on-chronic liver failure. J Hepatol 2014;61:1038-1047.

10 Pamecha V, Kumar S, Bharathy KGS: Liver transplantation in acute on chronic liver failure: challenges and an algorithm for patient selection and management. Hepatol Int 2015;9:534-542.

11 Finkenstedt A, Nachbaur K, Zoller H, et al: Acute-onchronic liver failure: excellent outcomes after liver transplantation but high mortality on the wait list. Liver Transpl 2013;19:879-886.

12 Artru F, Louvet A, Ruiz I, et al: Liver transplantation in the most severely ill cirrhotic patients: a multicenter study in acute-on-chronic liver failure grade 3. J Hepatol 2017;67:708-715.
3 Sharma P, Schaubel DE, Gong Q, et al: End-stage liver disease candidates at the highest model for end-stage liver disease scores have higher wait-list mortality than status-1A candidates. Hepatol Baltim Md 2012;55: 192-198.

14 Weismüller TJ, Fikatas P, Schmidt J, et al: Multicentric evaluation of model for end-stage liver disease-based allocation and survival after liver transplantation in Germany - limitations of the 'sickest first'-concept. Transpl Int 2011;24:91-99.

15 Duan B-W, Lu S-C, Wang M-L, et al: Liver transplantation in acute-on-chronic liver failure patients with high model for end-stage liver disease (MELD) scores: a single center experience of 100 consecutive cases. J Surg Res 2013;183:936-943.

16 Chan AC, Fan ST, Lo CM, et al: Liver transplantation for acute-on-chronic liver failure. Hepatol Int 2009;3: 571-581.

17 Bahirwani R, Shaked O, Bewtra M, et al: Acute-onchronic liver failure before liver transplantation: impact on posttransplant outcomes. Transplantation 2011;92:952-957. 